\title{
Reseña de la mesa redonda "La importancia de la Historia de la Educación como contenido para la formación de docentes", realizada en el Departamento de Investigaciones Educativas el 24 de noviembre de 2014
}

\author{
Aleida Garcia Aguirre \\ Departamento de Investigaciones \\ Educativas, Cinvestav \\ aleidagaguirre@gmail.com
}

En el marco de su Asamblea General, el VI Consejo Directivo de la Sociedad Mexicana de Historia de la Educación (SOMEHIDE) convocó a los socios y público en general a la mesa redonda "La importancia de la Historia de la Educación como contenido para la formación de docentes", el 24 de noviembre en las instalaciones del Departamento de Investigaciones Educativas del Cinvestav en la ciudad de México. A la mesa fueron invitados siete hombres y mujeres que hacen historia de la educación y que tienen relación estrecha con la formación de maestros; no obstante, por contratiempos personales, dos de los ponentes no asistieron. Los participantes fueron los siguientes: 1) Flor Marina Pérez López, de la Escuela Normal Superior de Maestros (ENSM); 2) Rusbelina Conzález Padilla, de la Escuela Normal "Profesor Serafín Peña", del estado de Nuevo León; 3) Sergio Ortiz Briano, de la Escuela Normal Rural de Cañada Honda, Aguascalientes; 4) Freddy Javier Espadas Sosa, de la Universidad Pedagógica Nacional (UPN), Unidad 31-A, Yucatán; y 5) Cirila Cervera Delgado, del Instituto de Investigaciones en Educación de la Universidad de Guanajuato.

De acuerdo con el contenido común de las intervenciones de la mesa, al hacer la invitación, el Consejo Directivo de la SOMEHIDE planteó dos preguntas concretas a los expositores: ¿Cómo se ha impartido la historia de la educación en las instituciones de formación docente?, ¿Por qué es importante que los docentes en formación conozcan la historia de la educación? y se sugirió la lectura del texto de Anne Marie Chartier, “¿Con qué historia de la educación debemos formar a los docentes?" (2008). ${ }^{1}$ En términos generales, la mesa

${ }^{1}$ El texto de Chartier fue redactado especialmente para presentarse en el Encuentro de la Sociedad Argentina de Historia de la Educación del 2008 
redonda dio a conocer el estado pasado y actual de la historia de la educación como contenido de la formación de docentes en diferentes entidades federativas y aportó valiosos elementos para pensar en el orden prescriptivo de la temática, ¿qué historia enseñar? y ¿quién debe enseñarla?

El punto de partida de la realización de la mesa redonda fue el reconocimiento pleno de la legitimidad del saber producido por la historia de la educación y, a partir de ahí, se estableció el debate respecto a cuáles deben ser las directrices de la transformación de ese saber en contenido curricular para la formación de docentes. El encuentro constituyó el primer acercamiento formal de la SOMEHIDE a esta cuestión y, por ende, las intervenciones de los ponentes han de ser comprendidas dentro del estado de gestación en que se encuentra la discusión.

A continuación dividiré esta reseña en tres apartados; en el primero expondré los temas transversales de las exposiciones; en el segundo destacaré las particularidades sobresalientes de algunas intervenciones; finalmente, aportaré elementos para continuar el debate a partir del convocado por la SOMEHIDE.

\section{Las temáticas transversales a las exposiciones ¿Cómo se ha impartido la historia de la educación en la formación de maestros?}

La mesa estuvo de acuerdo con Cervera Delgado en que la historia de la educación ha estado presente en los diferentes planes de estudio de formación docente, aunque ella ha reproducido los periodos e hitos de la historia nacional, ha privilegiado la historia política de la educación y, particularmente, de los triunfos alcanzados. Espadas Sosa formuló una crítica a esta forma de enseñar historia, y señaló que, en el contexto de lucha contra el neoliberalismo, la historia de la educación para la formación de docentes debe mantener una muy estrecha relación con la historia regional. Por su parte, Ortiz Briano introdujo el nivel institucional y asoció las fortalezas y deficiencias de la enseñanza de la historia de la educación con quienes integran la planta docente de las normales, y llamó la atención sobre las historias institucionales para comprender las particularidades en los contenidos y las evaluaciones. González Padilla, en un balance de los avances y retos que enfrenta la Escuela Normal "Profesor Serafín Peña" sobre la materia, apuntó que la institución y los profesores han enfatizado el método histórico y la producción historiográfica en la impartición de los tres cursos de historia de las licenciaturas de educación preescolar y primaria establecidos por la Reforma en Educación Normal 2012. 


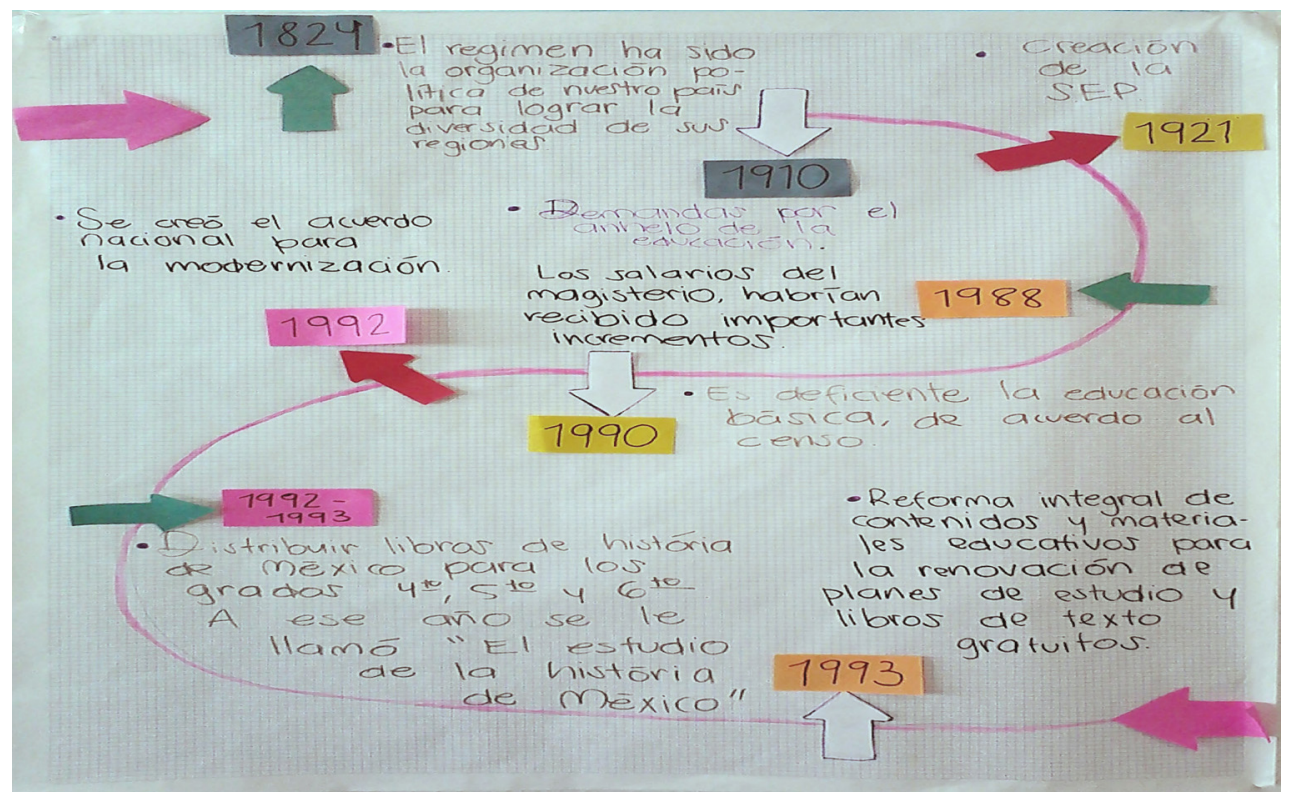

Imagen presentada por Cervera Delgado en la que se ilustra la representación de "historia de la educación" de un alumno. Obsérvense los saltos en el tiempo y los hitos que establece.

\section{¿Por qué debe impartirse historia de la educación en la formación de maestros?}

En este punto, las presentaciones tomaron dos caminos divergentes. Por un lado, González Padilla argumentó que la historia de la educación, lo mismo que los cursos de Educación Histórica en el Aula y en Diversos Contextos, deben estar dirigidos al desarrollo del pensamiento histórico de los normalistas y acercarlos al estudio de las fuentes primarias y la construcción de diferentes discursos de verdad histórica a partir del uso correcto de la metodología "[...] para posteriormente desarrollarla como maestros en las jornadas de observación práctica docente". Por otro lado, Espadas Sosa y Ortiz Briano enfatizaron la relación entre la construcción de la profesión docente y de la identidad del profesorado con el estudio de la historia de la educación. En conjunto, en la mesa redonda se pusieron a consideración dos aspectos importantes y relacionados del porqué de la enseñanza de la historia de la educación a futuros docentes y que deberán ser considerados para debates por venir: la conveniencia o no de que los normalistas construyan una historia de la educación original, utilizando la metodología y teoría de la historia y, al mismo tiempo, la pertinencia de que dicha historia (erigida o aprendida) se dirija hacia el proceso identitario sincrónico y diacrónico de los docentes. 


\section{¿Con qué historia de la educación formar a los docentes?}

En los apartados previos he señalado algunos elementos para desentrañar las posturas formuladas en la mesa respecto a cuál historia de la educación se debe aplicar a la hora de formar a los docentes. Cuando los ponentes abordaron las formas de enseñanza actuales y pasadas, también formularon prescripciones más o menos claras respecto al futuro deseable de la materia. Primero, la totalidad de los ponentes argumentó que la enseñanza memorística debe superarse para propiciar el pensamiento crítico sobre el pasado mediante la distinción de categorías claves de la disciplina; dicho pensamiento ha de dirigirse, de acuerdo con diferentes ponentes, hacia la creación de nueva historia o hacia el estudio de otras historiografías. Segundo, González Padilla, Ortiz Briano, Espadas Sosa y Cervera Delgado consideraron que la historia de la educación es un contenido curricular que debe impartir un profesional de la materia, es decir, alguien que conozca y sepa hacer historia de la educación de forma profesional. En ambas prescripciones, en la mesa se coincidió en que los investigadores y estudiosos de la historia de la educación y, por lo tanto, la SOMEHIDE y sus socios enfrentan la ardua tarea de elaborar propuestas de contenido y didáctica para la enseñanza de la historia de la educación a docentes; o en palabras de Ortiz Briano, el reto es que "[...] comunidades científicas como la nuestra, promuevan acercamientos hacia esas instituciones con la finalidad de facilitar la renovación de posturas e interpretaciones en torno a la enseñanza de la historia de la educación". Por otro lado, con argumentos de origen diverso, el grueso de los ponentes coincidió en que el conocimiento de historia de la educación "[...] sólo puede ayudar a formar docentes si tiene un valor de uso en la práctica del oficio de enseñante" (Espadas Sosa), en consonancia con la postura formulada por Chartier en el artículo citado (2008).

En suma, aunque la mesa no expresó un consenso definitivo respecto a los contenidos de la materia, al relacionar ésta con las discusiones académicas actuales sobre la historia y la historia de la educación, apuntaló una dirección hacia la cual orientar el debate.

\section{La coyuntura política que propicia el debate sobre la historia de la educación en la formación de docentes}

El conjunto de la mesa redonda señaló que la educación normal vive la antesala de una reforma profunda en la que es deseable que los investigadores y los formadores de docentes intervengan. Ortiz Briano y Espadas Sosa se refirieron al documento resultante del Foro Nacional para la revisión del modelo de Educación Normal, celebrado en Baja California Sur en junio del presente año, y en el cual se señala que la "recuperación histórica de la formación de maestros" debe ser uno de los ejes articuladores del nuevo modelo que defienda a las 
escuelas normales como "instituciones idóneas en la preparación de profesores". Si, como sugiere Ortiz Briano, los resultados de la educación normal están sometidos a un escrutinio estricto por algunos sectores del gobierno y los empresarios, el fortalecimiento de la historia de la educación en la formación de maestros, con el respaldo de profesores e investigadores, se traduciría no sólo en una fuente para la calidad del desempeño profesional de los futuros docentes, sino que sería uno de los fundamentos para la defensa de las normales del país.

\section{Particularidades sobresalientes de las intervenciones}

En esta sección me referiré a temáticas particulares abordadas por los ponentes de la mesa redonda; dichas cuestiones fueron seleccionadas por considerar que apuntan posibles líneas prospectivas de investigación y debate, no obstante el lector debe considerar que al mismo tiempo otros argumentos han quedado excluidos de esta reseña.

El trabajo de Ortiz Briano sobresalió por la investigación de archivo que realizó para hacer el recuento del contenido y la posición curricular del curso de historia de la educación (general y de México) en los planes de estudios de 1939, 1997 y 2012, deteniéndose en las traducciones concretas que realizaron tanto las instituciones como los profesores que impartieron los cursos; para este ponente fue importante descender del nivel de la historia de la educación de planes y programas hacia el nivel de la implantación de los mismos.

La intervención de González Padilla destacó por el balance de los objetivos y resultados de cada uno de los cursos de historia y las labores de rescate y conservación de objetos denominados "educativos" dentro de museos, así como de recuperación de memoria y testimonios de sujetos vinculados con la educación, en las que se involucran los normalistas.

Durante su intervención, Pérez López presentó opiniones de alumnos de la Escuela Nacional Superior de Maestros sobre la importancia de la historia de la educación en la currícula que desarrollan; en estas transcripciones se reveló que para los docentes en formación, la historia va ligada con la generación de pensamiento crítico y no repercute, como sugiere Anne Marie Chartier, en el trabajo docente. Sin duda alguna, las investigaciones futuras sobre la historia de la educación en el currículo de formación de profesores profundizarán en este tipo de indagaciones para comprender y explicar mejor los efectos que esta materia tiene en la formación y los primeros años de desempeño laboral.

La relación entre historia regional, historia de la educación y conformación de la identidad profesional fue abordada por Espadas Sosa en su presentación, quien además definió la importancia de que la elaboración de reformas considere la memoria histórica de los maestros en cada región del país. 
Por último, Cervera Delgado abrió el debate referente al análisis de la historia de la educación como contenido curricular en instituciones formadoras de docentes: ¿Acaso la historia de la educación, la historia de la pedagogía, la historia de las doctrinas pedagógicas y de la filosofía de la educación son equiparables? ¿Es deseable que la historia de la educación sea una hagiografía de profesores destacados, que únicamente responda a los hitos de la historia nacional? ¿Qué historia de la educación construir y cuál enseñar a los docentes en formación?

\section{Temas abiertos, preguntas pendientes}

La mesa fue un ejercicio enriquecedor para los historiadores de la educación y los formadores de maestros pues colocó a la SOMEHIDE en la urgencia de abrirse a otras instituciones y sectores sociales, aunque de hecho la propia Sociedad propició esta apertura al convocar un panel con la temática indicada. La hechura de la historia de la educación y la presentación de resultados en Encuentros que se sugirió durante el evento, no es suficiente; por el contrario, urge que en la coyuntura política actual y con la reforma de educación normal en puerta, los académicos y especialistas se congreguen para analizar y debatir la organización y el contenido curricular en la formación docente. En otros países de América Latina, Brasil y Argentina de forma destacada, los historiadores de la educación se han involucrado en el fortalecimiento de la historia de la educación en el currículo, han pugnado por que los docentes que impartan la materia sean los mismos historiadores de la educación y, por lo tanto, también han disputado que se generen nuevos espacios laborales para ellos mismos. Ahora bien, el involucramiento de la Sociedad en la definición de elementos del currículo conlleva el establecimiento de relaciones formales con instituciones estatales, y tanto la SOMEHIDE como los historiadores involucrados deberán ser cautelosos porque a la par de que pueden incidir en las reformas, su trabajo y perspectiva también pueden modificarse durante la interacción. Conviene que esta cuestión se discuta con rigor y se analicen, en forma paralela, las experiencias de otras latitudes.

En el multicitado texto, transversal a todas las exposiciones, Chartier relata las historias de la educación con las que se ha formado a los docentes en Francia y debate cuál es la historia de la educación que hoy debe enseñarse a los futuros docentes. Su conclusión es que los conocimientos que realmente forman a los docentes son aquellos que tienen "un valor de uso en la práctica del oficio" y, continúa, la historia de la educación que se integre al currículo debe tener como protagonistas a los profesores para generar una identidad profesional pero, sobre todo, para dotarlos de responsabilidad profesional. Las historias que "transforman el pasado en necesidad" y "'lo que tuvo lugar' en destino", las "teorías' sobre la escuela que predicen efectos deseables [...] o indeseables [...] suelen considerar a los 
educadores como una cuestión olvidable" y, en última instancia, sirven "de coartada para sus sentimientos de impotencia”. Para Anne Marie Chartier, la historia de la educación que debería formar a los docentes es aquella cercana a la "comprensión de la realidad —legítima en términos científicos - que presupone márgenes de indeterminación", que la historia no está concluída y que su actuación dentro del aula y en los márgenes de la profesión sí importa.

\section{Bibliografía}

Chartier, Anne Marie (2008), “¿Con qué historia de la educación debemos formar a los docentes?”, Historia de la Educación. Anuario, núm. 9, pp. 15-38.

Aleida García AcuirRe. Departamento de Investigaciones Educativas, Cinvestav. Maestra en Ciencias con la especialidad de Investigaciones Educativas, con interés particular en: Historia social de la educación (1930-1960); Historia del magisterio en México. Publicaciones recientes: Roldán Vera, Eugenia, Aleida García Aguirre y Marco Aurelio Vargas Morales (2013), "Liberté d'enseignement / libertad de enseñanza en el siglo XIX: trayectorias, variaciones y transferencias conceptuales entre México, Francia y Colombia", Proceedings of the 16th International Conference on the History of Concepts, Bilbao, pp. 184-193. García Aguirre, Aleida (2014), "Organización del gremio magisterial en la configuración histórica de movimientos sociales en México durante los años sesenta", en Alicia Civera Cerecedo, Carlos Escalante Fernández y Elsie Rockwell (comps.), Sujetos, poder y disputas por la educación, Zinacantepec, México, El Colegio Mexiquense, A. C., documento pdf en CD de datos. 